\title{
POSITIVE PERTURBATIONS OF UNBOUNDED OPERATORS
}

\author{
J. DOMBROWSKI
}

\begin{abstract}
This work studies the spectral properties of certain unbounded selfadjoint operators by considering positive perturbations of such operators and the unitary equivalence of the perturbed and unperturbed transformations. Conditions are obtained on the unitary operators implementing this equivalence which guarantee that the selfadjoint operators have an absolutely continuous part.
\end{abstract}

1. Introduction. Let $J$ be a selfadjoint operator defined on a dense subset $D_{J}$ of the Hilbert space $\mathcal{H}_{6}$. For $J=\int \lambda d E_{\lambda}$, denote by $\mathcal{H}_{a}(J)$ the set of elements $x$ in $\mathcal{H}$ for which $\left\|E_{\lambda} x\right\|^{2}$ is an absolutely continuous function of $\lambda$. It can be shown that $\mathcal{K}_{a}(J)$ is a subspace of $\mathcal{H}$ which reduces $J$. (See Halmos [1, p. 104]; Kato [2, p. 516].) The restriction of $J$ to $\mathscr{H}_{a}(J) \cap D_{J}$ is called the absolutely continuous part of $J$. If $\mathcal{H}_{a}(J)=\mathscr{H}$, the operator $J$ is said to be absolutely continuous. The absolutely continuous part of a unitary operator $U$, with spectral resolution $U=\int_{0}^{2 \pi} e^{i \lambda} d F_{\lambda}$, is defined in a similar way. Finally, for any operator $A$ on $\mathcal{H}$ let $\mathrm{sp}(A)$ denote the spectrum of $A$.

The subject of this paper is a selfadjoint operator $J$ which for some bounded nonnegative operator $D$ and unitary operator $U$ satisfies an equation of the form

$$
U J U^{*}=J+D \quad \text { with } \operatorname{sp}(U) \neq\{z:|z|=1\},
$$

so that $J$ is unitarily equivalent to $J+D$. Under the assumption that for some real constant $c, J \geqslant c I$ or $J \leqslant c I$, it has been shown by Putnam [3, Theorem 2.12 .2$, p. 38] that $J$ has an absolutely continuous part. Also, if $J$ is unbounded and $(-\infty, \infty)-\operatorname{sp}(J)$ contains an open interval of length greater than $\|D\|$, then $J$ must have an absolutely continuous part (see Putnam [3]). It is the purpose of this paper to consider an unbounded selfadjoint operator $J$ satisfying (1.1) with no restrictions on the spectrum of $J$. Appropriate examples will be presented. This work was motivated by the questions posed in Putnam [4].

2. Main result. The main result to be established is as follows.

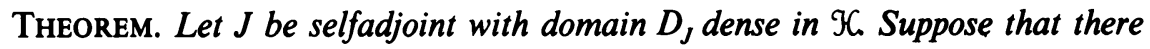
exists $a$ unitary operator $U$ and a bounded operator $D \geqslant 0, D \neq 0$, satisfying

Received by the editors September 16, 1976.

AMS (MOS) subject classifications (1970). Primary 47B25.

- American Mathematical Society 1977 
(1.1). If for some real $\theta$ for which $e^{i \theta} \notin \mathrm{sp}(U),\left(I-e^{i \theta} U^{*}\right)^{-1}\left(D_{J}\right) \subset D_{J}$, then $J$ has an absolutely continuous part.

Proof. Let $U_{\theta}=e^{-i \theta} U$. Since $e^{i \theta} \notin \operatorname{sp}(U), 1 \notin \operatorname{sp}\left(U_{\theta}\right)$. Therefore $U_{\theta}$ is the Cayley transform of a bounded selfadjoint operator $Q$. In particular,

$$
U_{\theta}=(Q-i I)(Q+i I)^{-1}=\left[I-2 i(Q+i I)^{-1}\right] .
$$

For $x$ in $D_{J}, U_{\theta} J U_{\theta}^{*} x=J x+D x$ and so $J U_{\theta}^{*} x=U_{\theta}^{*} J x+U_{\theta}^{*} D x$. It then follows that

$$
J\left[I+2 i(Q-i I)^{-1}\right] x=\left[I+2 i(Q-i I)^{-1}\right] J x+U_{\theta}^{*} D x,
$$

or equivalently, that

$$
J(Q-i I)^{-1} x=(Q-i I)^{-1} J x-\frac{1}{2} i U_{\theta}^{*} D x .
$$

If $C$ is defined by $D=2(Q+i I)^{-1} C(Q-i I)^{-1}$, then

$$
J(Q-i I)^{-1} x=(Q-i I)^{-1} J x-i(Q-i I)^{-1} C(Q-i I)^{-1} x .
$$

For $x$ in $D_{J}$, let $y=(Q-i I)^{-1} x$. Then $x=(Q-i I) y$ and

$$
(Q-i I) J y=J(Q-i I) y-i C y .
$$

It follows immediately that

$$
Q J=J Q-i C \quad \text { on }(Q-i I)^{-1}\left(D_{J}\right) .
$$

Note that since $U_{\theta}^{*}=I+2 i(Q-i I)^{-1}$ and $U_{\theta}^{*}\left(D_{J}\right) \subset D_{J},(Q-i I)^{-1}\left(D_{J}\right)$ $\subset D_{J}$.

For $y$ in $(Q-i I)^{-1}\left(D_{J}\right)$ let $z=(J-i I) y$. Then $y=(J-i I)^{-1} z$ and

$$
(J-i I)^{-1} Q z=Q(J-i I)^{-1} z-i(J-i I)^{-1} C(J-i I)^{-1} z .
$$

It then follows that

$$
\left[I+2 i(J-i I)^{-1}\right] Q z=Q\left[I+2 i(J-i I)^{-1}\right] z+2(J-i I)^{-1} C(J-i I)^{-1} z .
$$

Let $V$ denote the Cayley transform of $J$ so that

$$
V=(J-i I)(J+i I)^{-1}=\left[I-2 i(J+i I)^{-1}\right] .
$$

Then

$$
V^{*} Q z=Q V^{*} z+2(J-i I)^{-1} C(J-i I)^{-1} z
$$

Hence

(2.1) $V Q V^{*}-Q=-2(J+i I)^{-1} C(J-i I)^{-1}$ on $(J-i I)(Q-i I)^{-1}\left(D_{J}\right)$. 
Since, as noted above, $(Q-i I)^{-1}\left(D_{J}\right) \subset D_{J}$ it follows that $(Q-i I)\left(D_{J}\right)$ $\supset D_{J}$. But

$$
Q=i\left(I+U_{\theta}\right)\left(I-U_{\theta}\right)^{-1}=-i\left(I+U_{\theta}^{*}\right)\left(I-U_{\theta}^{*}\right)^{-1}
$$

and, by hypothesis, $D_{J}$ is invariant under $U_{\theta}^{*}$ and $\left(I-U_{\theta}^{*}\right)^{-1}$. Therefore $D_{J}$ is invariant under $Q$ and hence under $(Q-i I)$. Thus $(Q-i I) D_{J}=D_{J}$ and $(J-i I)(Q-i I)^{-1} D_{J}=\mathcal{H}$.

It has been shown, therefore, that the equation

$$
V Q V^{*}-Q=-2(J+i I)^{-1} C(J-i I)^{-1}
$$

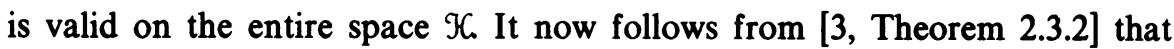
$\mathcal{K}_{a}(V)=\mathcal{K}_{a}(J)$ contains the least subspace of $\mathcal{K}$ reducing $V$ and containing the range of $-2(J+i I)^{-1} C(J-i I)^{-1}$, which completes the proof.

COROllaRY. If, in addition to the conditions imposed on $J, U$ and $D$ in the above Theorem, 0 is not in the point spectrum of $D$, then $J$ is absolutely continuous.

REMARK. The condition $\left(I-e^{i \theta} U^{*}\right)^{-1}\left(D_{J}\right) \subset D_{J}$ is used to show that the set $(J-i I)(Q-i I)^{-1}\left(D_{J}\right)$ in $(2.1)$ is dense in $\mathcal{G}$, a fact which is not obvious if $J$ is unbounded. That the condition is not that restrictive will be illustrated by the following example.

3. An example. Consider $\mathscr{K}=L^{2}(-\infty, \infty)$. Let $P=-i d / d x$ and choose $m(x)$ to be a real valued measurable function on $(-\infty, \infty)$ with the property that $J=P+m$ on $D_{J}=\left\{f \in L^{2}(-\infty, \infty): f\right.$ absolutely continuous, $-i f^{\prime}$ $\left.+m(x) f(x) \in L^{2}(-\infty, \infty)\right\}$ is selfadjoint. [It is sufficient, for example, that $m(x)$ be bounded.]

Let $q(x)$ be a real valued, positive, measurable function on $(-\infty, \infty)$ which is bounded above. If $D$ denotes the selfadjoint operator on $\mathcal{H}$ corresponding to multiplication by $q(x)$ then $D$ is nonnegative and bounded. Let $U$ denote the unitary operator on $\mathcal{H}$ corresponding to multiplication by $e^{-i \phi(x)}$ where $\phi(x)=\int_{0}^{x} q(t) d t$. Then $U J U^{*}=J+D$. (See Kato [2, pp. 528-529].) Also, as noted in Putnam [4], a similar argument shows that for any real constant $c, P+m+c$ is unitarily equivalent to $P+m$. Hence $\operatorname{sp}(J)=(-\infty, \infty)$. Finally, if $q(x)$ is chosen so that $\int_{-\infty}^{\infty} q(t) d t<\pi$, then $\operatorname{sp}(U) \neq\{z:|z|=1\}$. The aim now is to show that $J$ is absolutely continuous.

For some real $\theta, e^{i \theta} \notin \operatorname{sp}(U)$. Hence $\left(I-e^{i \theta} U^{*}\right)^{-1}$ exists and, in fact, corresponds to multiplication by $\left\{1-e^{i[\theta+\phi(x)]}\right\}^{-1}$ where, as defined above, $\phi(x)=\int_{0}^{x} q(t) d t$. To show that $\left(I-e^{i \theta} U^{*}\right)^{-1}\left(D_{J}\right) \subset D_{J}$, choose $f$ in $D_{J}$ and let $g=\left(I-e^{i \theta} U^{*}\right)^{-1} f$. Since $\left(I-e^{i \theta} U^{*}\right)^{-1}$ is a bounded operator on $\mathcal{H}$ it is clear that $g$ is in $L^{2}(-\infty, \infty)$. Furthermore, $g(x)=\chi(x) f(x)$ where $\chi(x)$ $=\left\{1-e^{i[\theta+\phi(x)]}\right\}^{-1}$. Thus $g$ is a product of absolutely continuous functions and hence is itself absolutely continuous. Finally, a direct computation shows that $-i g^{\prime}+m g=\left[-i f^{\prime}+m f\right]-i \chi^{\prime} f$. Since $f$ is in $D_{J}$ and $\chi$ and $\chi^{\prime}$ are bounded, it follows that $-i g^{\prime}+m g$ is in $L^{2}(-\infty, \infty)$ and hence that $g$ is in $D_{J}$. 
The Theorem of $\$ 2$ shows, therefore, that if $J=P+m$ is selfadjoint, then $J$ has an absolutely continuous part. Moreover, since 0 is not in the point spectrum of $D, J$ is absolutely continuous.

REMARKs. That $J=P+m$ is absolutely continuous can also be established as follows. If $m$ is locally integrable on $(-\infty, \infty)$ it is a consequence of [5, Theorem 10.6] that $J$ is unitarily equivalent to $P$ and hence absolutely continuous. Moreover, as observed by the referee, it can be shown that $m$ must be locally integrable on $(-\infty, \infty)$ for $J$ to be selfadjoint.

Finally, it is perhaps worth noting that operator equations of the form $U_{t} A U_{t}^{*}=A+D(-\infty<t<\infty)$ with $A$ selfadjoint, $D$ nonnegative, and $U_{t}=e^{i t H}$ for some fixed selfadjoint operator $H$, do occur in quantum mechanics. Here $A$ represents an observable on a quantum mechanical system with Hamiltonian $H$, which does not explicitly depend on time. In case $A$ is bounded or half-bounded several results are given in Putnam [3, pp. 40-41]. (See also the references cited there.) It seems that further investigations in this direction could lead to some interesting applications.

\section{REFERENCES}

1. P. R. Halmos, Introduction to Hilbert space and the theory of spectral multiplicity, Chelsea, New York, 1951. MR 13, 563.

2. T. Kato, Perturbation theory for linear operators, Springer-Verlag, New York, 1966. MR 34 \#3324.

3. C. R. Putnam, Commutation properties of Hilbert space operators and related topics, SpringerVerlag, New York, 1967. MR 36 \# 707.

4. - Positive perturbations and unitary equivalence, Canad. J. Math. 29 (1977), 161-164.

5. M. Stone, Linear transformations in Hilbert space and their applications to analysis, Amer. Math. Soc. Colloq. Publ., vol. 15, Amer. Math. Soc., Providence, R.I., 1932.

Department of Mathematics, Wright State University, Dayton, Ohio 45431 